\title{
Análise do Desempenho Motor associado ao Estado Nutricional de Idosos cadastrados no Programa Saúde da Família, no município de Vitória de Santo Antão-PE
}

\author{
Analysis of Motor Performance associated with the Nutritional \\ Status of the Elderly enrolled in the Family Health Program in the \\ municipality of Vitória de Santo Antão in the State of Pernambuco
}

\author{
Lisandra Delfino de Albuquerque Soares ${ }^{1}$ \\ Florisbela de Arruda Câmara e Siqueira Campos ${ }^{1}$ \\ Maria das Graças Rodrigues de Araújo ${ }^{1}$ \\ Ana Patrícia Siqueira Tavares Falcão ${ }^{2}$ \\ Bruna Rafaela Dornelas de Andrade Lima ${ }^{1}$ \\ Danielle Ferreira de Siqueira ${ }^{1}$ \\ Etiene Oliveira da Silva Fittipaldi ${ }^{1}$ \\ Silvana Gonçalves Brito de Arruda ${ }^{1}$ \\ Zelyta Pinheiro de Faro ${ }^{1}$
}

${ }^{1}$ Núcleo de Educação Física e Ciências do Esporte, Centro Acadêmico de Vitória, Universidade Federal de Pernambuco. Rua Sylla Pessoa de Melo 18, São

Vicente de Paulo. 55604-125 Vitória de Santo Antão PE.

dlisandra@hotmail.com

${ }^{2}$ Instituto Federal de

Educação, Ciência e

Tecnologia.

\begin{abstract}
The scope of this paper was to investigate the relationship between the nutritional status and the motor skills of the elderly registered in the Family Health Program in the urban area of Vitória de Santo Antão in the State of Pernambuco, by means of a transversal epistemological descriptive-analytical field study. The participants of this survey were 235 elders, both male and female of 60-years-old and over registered in the family health program in the urban area of the city. The nutritional status was evaluated by the body mass index and the circumference of the calf. Four motor tests were used to evaluate motor performance: Time Up\&Go, Tinetti's Index, Functional Reach and Unipodal Support. The associations were checked using Spearman's correlation. The association of the nutritional status with the motor tests was statistically significant among males $^{*}$ or females ${ }^{* *}$ : (Time Up\&Go rho $=0.290^{*}$ / 350**; Functional Reach rho $=0.232^{*} / 352^{* *}$; Tinetti's Index rho $=243^{*} / 363^{* *}$ and Unipodal Support rho $=221^{*} / 475^{* *}$. The evaluation of the results revealed an association between nutritional status and functional capacity of the elders no matter what gender; with obesity being a limiting nutritional condition to adequate performance in the tests.
\end{abstract}

Key words Geriatric assessment, Elderly nutrition, Physical activity
Resumo Investigar a associação entre o estado nutricional, e o desempenho das habilidades motoras, de idosos, cadastrados no Programa Saúde da Família, zona urbana, do município de Vitória de Santo Antão (PE). Estudo epidemiológico, transversal de campo do tipo descritivo-analítico. Participaram desta pesquisa 235 idosos com idade superior ou igual a 60 anos, do gênero masculino e feminino, residentes em Vitória (PE) e cadastrados nas unidades de saúde da família da zona urbana da cidade. O estado nutricional foi avaliado segundo o Índice de Massa Corporal e a Circunferência de Panturrilha. Para análise do desempenho motor foram utilizados quatro testes motores: Time Up\&Go, Indice de Tinetti, Alcance Funcional e Apoio Unipodal. As associações foram verificadas segundo a correlação de Spearman. A associação do estado nutricional com os testes motores foi estatisticamente significativa entre os gêneros masculino $^{*}$ e feminino ${ }^{* *}$ (time up\&go rho $=$ $0,290^{*}, 350^{* *}$, alcance funcional rho $=0,232^{*}$, $352^{* *}$, indice de Tinetti rho $=243^{\star}, 363^{* *}$ e apoio unipodal rho $=221^{*}, 475^{* *}$ ) não apresentando distinção entre sexo. A avaliação dos resultados mostrou associação entre o estado nutricional e a capacidade funcional entre os idosos independente do sexo; tendo sido a obesidade apontada como a condição nutricional limitante ao desempenho adequado nos testes.

Palavras-chave Avaliação geriátrica, Nutrição do idoso, Atividade física 


\section{Introdução}

O processo de envelhecimento vem atingindo níveis mundiais. Este processo vem ocorrendo de forma bastante acelerada no Brasil, decorrente principalmente da queda da fecundidade observada a partir do final dos anos $60^{1-3}$. Indicadores da Organização Mundial de Saúde (OMS) informam que o Brasil em 2025 será o sexto país do mundo em número de idosos, totalizando 30 milhões de pessoas $^{1,4,5}$. Associado a isto o Brasil vem apresentando um aumento significativo na expectativa de vida da população ${ }^{6,7}$, que nos últimos 50 anos teve um acréscimo de aproximadamente 17,8 anos $^{6}$.

Esta transição demográfica exige mudanças qualitativas das formas de organização da sociedade, ao mesmo tempo em que serve de marco para mudanças culturais e econômicas que devem acompanhar este processo ${ }^{8}$.

O envelhecimento traz consigo uma série de alterações tanto na composição corporal, como mudanças orgânico-funcionais. Estas alterações são gradativas e gerais, podendo ser verificadas em idades variadas e em menor ou maior grau a depender das características genéticas de cada um. Sendo também um processo universal e irreversível, que se acelera na maturidade e provoca uma perda progressiva no organismo $0^{9,10}$.

Uma importante alteração do envelhecimento é a redução na força muscular que ocorre aparentemente ao mesmo tempo em que há uma diminuição das fibras musculares e, consequentemente, da massa muscular durante o final da meia idade e dos anos posteriores da idade adulta. Segundo Gallahue e Ozmun em 2002 $2^{11}$, por volta dos sessentas anos há uma perda aproximada de $20 \%$ da força muscular, chegando até a $40 \%$ entre os 70 e 80 anos. Podem ser observadas também alterações no sistema cardíaco, nervoso, respiratório e nos cinco sentidos com o processo de envelhecimento ${ }^{9,11,12}$.

Com a redução das fibras musculares ocorrerá o comprometimento do desempenho neuromuscular, evidenciado pela fraqueza muscular, pela lentidão dos movimentos, pela perda da força muscular e pela fadiga muscular precoce, constituindo um aspecto marcante do envelhecimento, e trazendo como consequência limitações funcionais para caminhar, levantar-se, e manter o equilíbrio postural, assim como, quedas iminentes. Estas limitações levam a dificuldades na execução das atividades da vida diária (AVD), à dependência funcional e à incapacidade ${ }^{13}$.

Alterações no estado nutricional (EN) também são observadas com as mudanças em que o organismo é submetido durante o envelhecimento, e a inadequação nutricional afeta o bem-estar de longevos, causando declínio funcional, devido aos aportes deficitários de calorias e nutrientes (desnutrição calórico-protéica, deficiência de vitaminas e minerais), pelo excesso calórico (obesidade) ou pela utilização excessiva de substâncias como o álcool ${ }^{5}$.

A inadequação nutricional leva a várias consequências, dentre elas pode-se citar as alterações na capacidade de realizar as $\mathrm{AVD}^{14-16}$.

Investigar sobre as incapacidades funcionais bem como sua relação com o EN em pessoas idosas é necessário para entender como os idosos estão aproveitando os anos adicionais ganhos com o aumento da expectativa de vida. Nos países em que o processo de envelhecimento populacional já não é um fenômeno recente há um maior conhecimento sobre os padrões de limitação funcional entre os idosos, porém no Brasil, ainda existem poucos estudos sobre esse tema ${ }^{17}$.

\section{Metodologia}

Estudo epidemiológico, transversal de campo do tipo descritivo-analítico. O presente foi realizado na cidade de Vitória de Santo Antão, localizada na zona da Mata Sul do estado de Pernambuco, distante $55 \mathrm{~km}$ da cidade do Recife. A coleta foi realizada no período de março à setembro de 2010, nas 20 unidades do Programa Saúde da Família (PSF) da zona urbana da cidade (Santana, Caiçara, Conceição, Cidade de Deus, Lagoa Redonda, Doutor Alvinho, Lídia Queiroz, Maranhão, Redenção, Amparo, Jardim Ipiranga, Maués, Alto José Leal, PACS Urbano I, PACS Urbano II, Matadouro, Mário Bezerra, Cajueiro, Bela Vista I e Bela Vista II) conforme dados do Sistema de Informação Básica (SIAB).

Participaram desta pesquisa 235 idosos com idade superior ou igual a 60 anos, dos gêneros masculino e feminino, residentes em Vitória-PE e cadastrados nas Unidade de Saúde da Família referentes a área de abrangência de sua residência, da zona urbana da cidade. Foram excluídos do projeto idosos com: demência senil; doença ou sequela neurológica que interfira na capacidade de equilíbrio corporal; doenças ortopédicas que interfira nas AVD ou na locomoção; cegueira ou déficit visual grave que interfira na locomoção independente; portadores de órteses ou dependentes de cadeiras de rodas; e, hipertensão arterial sistêmica moderada ou grave à hora do exame ${ }^{18}$.

Os idosos foram submetidos a uma avaliação do EN e de desempenho motor (DM), realizados 
por fisioterapeutas e estudantes do curso de graduação em nutrição, previamente capacitados.

$\mathrm{O}$ peso (em $\mathrm{kg}$ ) foi mensurado por meio de uma balança digital portátil da com capacidade para $150 \mathrm{~kg}$ e sensibilidade de $50 \mathrm{~g}$. Para aferição do peso, o idoso estava descalço e com vestimentas leves. A altura (em metros) foi aferida com o auxílio de um estadiômetro de parede. Para obtenção da altura, o idoso foi posicionado, com os pés lado a lado, encostando calcanhares, nádegas, omoplatas e parte posterior da cabeça na régua do estadiômetro ${ }^{19}$. As medidas do peso e da altura foram realizadas em triplicata e a média dos valores de cada uma delas foi utilizada para as análises.

Para a medição da circunferência da panturrilha, foi utilizada uma fita métrica, de plástico, inelástica, na superfície da pele, de modo que ficasse esticada, porém não apertada. A medida foi realizada com os indivíduos em pé, e a fita métrica foi posicionada horizontalmente na área de maior diâmetro da panturrilha, no ponto médio entre a cabeça da fíbula e o maléolo lateral $^{20}$. A avaliação do EN dos idosos foi verificada pelo índice de massa corporal [IMC $=\mathrm{MC}(\mathrm{kg})$ / Estatura $\left(\mathrm{m}^{2}\right)$ ] e pela circunferência da panturrilha. Para a análise do IMC foi utilizada a seguinte classificação ${ }^{21}: \leq 22=$ baixo peso; $>22$ e $<27=$ adequado; e $\geq 27$ = sobrepeso. Para a circunferência de panturrilha foi utilizado o ponto de corte de $30,5 \mathrm{~cm}$ para ambos os gêneros ${ }^{20}$.

A análise do desempenho motor consistiu de quatro testes (Time up\&go-TUG, Índice de Tinetti-IT, Alcance Funcional-AF e Apoio Unipodal-AU) que avaliavam entre outros o equilíbrio, mobilidade e flexibilidade. Durante o TUG foi mensurado em segundos, o tempo que o idoso levava para levantar da cadeira, andar uma distância de $3 \mathrm{~m}$, dar a volta, caminhar de volta e sentar na cadeira ${ }^{22}$; representando, desta forma, um conjunto de atividades rotineiras importantes para a independência funcional. Os idosos realizavam o teste uma vez, para compreendê-lo, e só na segunda tentativa o tempo era cronometrado.

A utilização do IT consistiu de duas escalas: de equilíbrio e de marcha. A primeira possui 09 itens: equilíbrio sentado, levantando, tentativas de levantar, assim que levanta, equilíbrio em pé, teste dos três tempos, olhos fechados, girando $360^{\circ} \mathrm{e}$ sentando. A segunda possui 07: início da marcha, comprimento e altura dos passos, simetria dos passos, continuidade dos passos, direção, tronco e distância dos tornozelos. A pontuação total do índice é de 28 pontos. Pontuação menor que 19 indica risco cinco vezes maior de quedas ${ }^{8,23}$.
O teste AF ("Functional Reach Test") ou "teste do alongamento funcional" foi desenvolvido por Duncan et al. ${ }^{24}$. Uma fita métrica é fixada à parede, na altura do ombro do paciente, que fica em pé na posição de lado em relação à parede, com os pés separados e alinhados em relação aos ombros e com os braços elevados a 90 graus de flexão, com extensão de punho e dedos. O paciente será instruído a alongar o corpo para frente o máximo possível, sem movimentar os pés, sem perder o equilíbrio, sem dar um passo e sem tocar na parede ou na fita. São realizadas três medições, e o resultado final é a média. Deslocamentos menores que $15 \mathrm{~cm}$ indicam risco de quedas.

$\mathrm{O}$ teste $\mathrm{AU}$ analisa o tempo que os idosos conseguem permanecer em apoio unipodal, com os olhos abertos e com os olhos fechados, avaliando deste modo seu equilíbrio; tempos inferiores a 5 segundos sugerem maior probabilidade de quedas ${ }^{25}$.

Este trabalho foi realizado respeitando os aspectos éticos, aplicando-se o Termo de Consentimento Livre e Esclarecido. O projeto de pesquisa foi submetido e aprovado pelo Comitê de Ética em Pesquisa envolvendo seres humanos do Centro de Ciências da Saúde da Universidade Federal de Pernambuco (CEP/CCS/UFPE).

Quanto ao método estatístico, foi realizada inicialmente uma análise descritiva por meio de mediana, valores míninos e máximos, e, uma distribuição de frequência. Posteriormente, foi realizada uma correlação de Spearman entre os indicadores nutricionais e os testes de desempenho motores. Todas as análises foram realizadas no Programa SPSS versão 18.0. A margem de erro (ou nível de significância) utilizado na decisão dos testes estatísticos foi de 5,0\%.

\section{Resultados}

A idade dos idosos variou de 60 a 94 anos, 69,09 $\pm 7,134$ anos. Para o sexo feminino $(\mathrm{n}=183)$, a média etária foi de 67,84 \pm 6,07 anos (60 a 84 anos). Para o sexo masculino $(\mathrm{n}=52)$ foi de 73,5 $\pm 8,74$ anos (60 a 94 anos).

$\mathrm{Na}$ Tabela 1 observa-se a distribuição dos idosos, segundo sexo, grupo etário e IMC. Verifica-se um predomínio da obesidade em ambos os sexos e faixas etárias.

A Tabela 2 apresenta os idosos distribuídos de acordo com seu desempenho nos testes TUG e AF, verifica-se um aumento do número de idosos com comprometimento funcional e com risco de quedas com o avançar da idade. 
A Correlação de Spearman's apresentou uma associação significativa entre o IMC e os testes de $\mathrm{DM}$, independente do gênero. Como pode ser visto na Tabela 3.

Quando analisados todos os idosos (235), as associações feitas pela correlação de Spearman's entre a CP e os testes de DM também foram significativas.

No Gráfico 1 é possível observar que idosos com sobrepeso apresentam maior comprometimento funcional, independentes do sexo, quando comparados aos com peso normal e baixo peso.

O Gráfico 2 refere-se a uma proporção entre a CP e o teste de alcance funcional, o qual avalia risco de quedas em idosos, percebe-se que quando a CP encontra-se acima de 30,5 cm (CP deficiente), a proporção de idosos com elevado risco de quedas aumenta, para ambos os gêneros.

Tabela 1. Distribuição dos Idosos segundo grupo etário, sexo e IMC

\begin{tabular}{|c|c|c|c|c|c|c|}
\hline \multirow[b]{2}{*}{ IMC } & \multicolumn{2}{|c|}{$\begin{array}{c}60-69 \\
\text { anos }\end{array}$} & \multicolumn{2}{|c|}{$\begin{array}{c}70-79 \\
\text { anos }\end{array}$} & \multicolumn{2}{|c|}{$\begin{array}{l}\geq 80 \\
\text { anos }\end{array}$} \\
\hline & $\mathbf{N}$ & $\%$ & $\mathbf{N}$ & $\%$ & $\mathbf{N}$ & $\%$ \\
\hline \multicolumn{7}{|l|}{ Feminino } \\
\hline Baixo Peso & 8 & 8,1 & 2 & 2,9 & 1 & 6,3 \\
\hline Peso Normal & 21 & 21,2 & 22 & 32,4 & 2 & 12,5 \\
\hline Obesidade & 70 & 70,7 & 44 & 64,7 & 13 & 81,3 \\
\hline Total & 99 & 100 & 68 & 100 & 16 & 100 \\
\hline \multicolumn{7}{|l|}{ Masculino } \\
\hline Baixo Peso & 2 & 10 & 3 & 16,7 & 1 & 7,1 \\
\hline Peso Normal & 6 & 30 & 5 & 27,8 & 6 & 42,9 \\
\hline Obesidade & 12 & 60 & 10 & 55,6 & 7 & 50 \\
\hline Total & 20 & 100 & 18 & 100 & 14 & 100 \\
\hline
\end{tabular}

Tabela 3. Correlação de Spearman's entre IMC e testes motores em idosos

\begin{tabular}{lcccc}
\hline & $\begin{array}{c}\text { Time } \\
\text { up\&go }\end{array}$ & $\begin{array}{c}\text { Alcance } \\
\text { funcional }\end{array}$ & $\begin{array}{c}\text { Índice de } \\
\text { Tinetti }\end{array}$ & $\begin{array}{c}\text { Apoio } \\
\text { Unipodal }\end{array}$ \\
\cline { 2 - 5 } & rho & rho & rho & rho \\
\hline Masculino & $0,290^{\star}$ & $0,232^{\star}$ & 0,243 & $0,221^{\star}$ \\
Feminino & $0,350^{*}$ & $0,352^{\star}$ & $0,363^{\star}$ & $0,475^{\star}$ \\
\hline
\end{tabular}

*rho $(\mathrm{p}<0,05)$

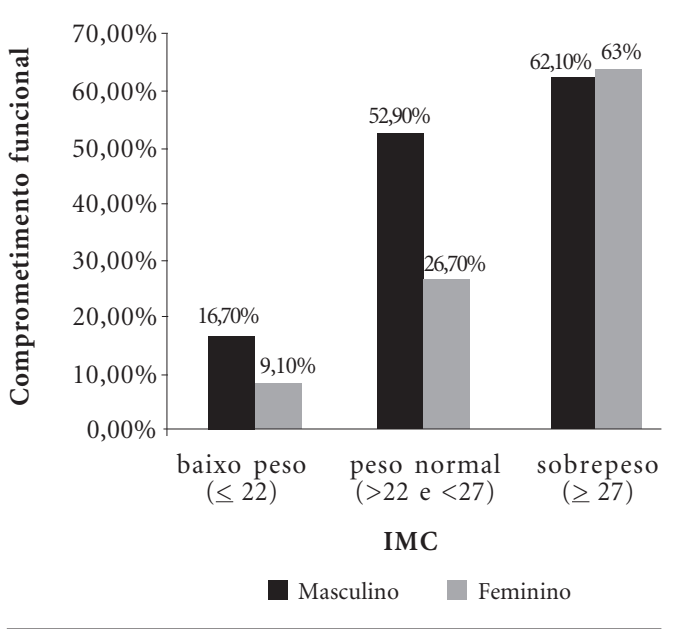

Gráfico 1. Associação entre IMC e comprometimento funcional (TUG) de idosos cadastrados no PSF Vitória/PE.

Tabela 2. Distribuição dos Idosos segundo grupo etário, Time Up\&Go e Alcance Funcional

\begin{tabular}{|c|c|c|c|c|c|c|}
\hline & \multicolumn{2}{|c|}{ 60-69 anos } & \multicolumn{2}{|c|}{ 70-79 anos } & \multicolumn{2}{|c|}{$\geq 80$ anos } \\
\hline & $\mathbf{N}$ & $\%$ & $\mathbf{N}$ & $\%$ & $\mathbf{N}$ & $\%$ \\
\hline \multicolumn{7}{|l|}{ Time Up\&Go } \\
\hline Adultos Saudáveis & 11 & 9,2 & 5 & 5,8 & 0 & 0 \\
\hline Normal para idosos & 52 & 43,7 & 37 & 43 & 9 & 30 \\
\hline Comprometimento Funcional & 56 & 45,4 & 44 & 51,2 & 21 & 30 \\
\hline Total & 119 & 100 & 86 & 100 & 30 & 100 \\
\hline \multicolumn{7}{|l|}{ Alcance Funcional } \\
\hline Sem risco & 11 & 9,2 & 12 & 14 & 1 & 3,3 \\
\hline duas vezes risco de cair & 54 & 45,4 & 33 & 38,4 & 10 & 33,3 \\
\hline quatro vezes risco de cair & 54 & 45,4 & 41 & 47,7 & 19 & 63,3 \\
\hline Total & 119 & 100 & 86 & 100 & 30 & 100 \\
\hline
\end{tabular}




\section{Discussão}

O envelhecimento populacional é preocupação mundial, devido à predisposição da população idosa a sofrer com os sinais e sintomas das comorbidades crônico-degenerativas, as quais costumam atingir em maiores proporções esta população gerando uma atenção especial no seu tratamento. O controle dos principais problemas de saúde que atingem os indivíduos idosos pode proporcionar a estes, além de vida mais longa e produtiva, uma melhor qualidade desta ${ }^{12}$.

Os resultados do presente estudo mostraram associação entre estado nutricional (IMC, CP) e desempenho nos testes motores. Foi observado que idosos com obesidade apresentam menor percentual de indivíduos com as capacidades funcionais adequadas, e levaram mais tempo para completar a caminhada de $3 \mathrm{~m}$ do Time Up\&Go, quando comparados aos eutróficos e aos de baixo peso.

Vários fatores podem influenciar a associação entre a obesidade e o baixo desempenho nos testes motores. A primeira relaciona-se ao maior risco e gravidade da osteoartrite nos joelhos ${ }^{26}$; o excesso de gordura aumenta a sobrecarga corporal, dificultando os movimentos e aumentando o estresse nas articulações e músculos, favorecendo, desta maneira, ao maior grau de incapacidade nos idosos obesos ${ }^{19,27,28}$.

No presente estudo $66,4 \%$ dos idosos apresentaram sobrepeso, estes resultados corroboram com os dados apresentados por levantamentos nacionais que vem indicando uma tendência para excesso de peso neste grupo populacional ${ }^{29,30}$. Lebrão e Laurenti ${ }^{31}$, em 2005, também encontraram elevada prevalência de excesso de peso em mulheres idosas (40.5\%).

A obesidade, dentre outros fatores, encontra-se associada à inatividade física, que pode levar ao baixo condicionamento físico (musculoesquelético e cardiorrespiratório), aumentando a fragilidade do idoso, e podendo deixá-lo mais vulnerável a doenças crônicas ${ }^{25}$. Em contrapartida uma incapacidade preexistente pode reduzir a atividade física e, consequentemente, aumentar o seu peso corporal, devido à redução do gasto energético ${ }^{30}$.

Estudos comprovam que existe uma relação de diminuição da aptidão funcional com o avanço da idade ${ }^{32,33}$. No presente estudo, $51,5 \%$ dos idosos avaliados apresentaram comprometimento funcional, analisados através do time Up\&Go e $89,8 \%$ apresentavam risco de quedas (Tabela 2).

Estes resultados estão de acordo com os dados apresentados por outras pesquisas que indicam um baixo nível de desempenho físico em idosos. Rogatto e Gobbi ${ }^{34}$, em 2001, realizaram um estudo comparativo entre mulheres jovens e idosas e observaram que o envelhecimento tem relação com uma diminuição da força muscular em idosos, mesmo com a prática regular de atividade física. Esta diminuição de força muscular relaciona-se com uma maior probabilidade de quedas em idosos ${ }^{35}$.

$\mathrm{Na}$ literatura, poucos foram os estudos que investigaram a associação do EN e testes que avaliam desempenho motor. Alguns artigos que verificaram esta associação divergem em alguns aspectos metodológicos do presente estudo, o que dificulta a comparação dos resultados. No trabalho realizado por Barbosa et al. ${ }^{29}$, em 2007, foi constatado que idosas com excesso de peso eram mais lentas na realização dos testes motores, quando comparadas às de peso normal.

No estudo realizado por Zuliane et al. ${ }^{36}$, em 2001, em Pádua, Norte da Itália, 98 indivíduos de 65 anos ou mais, de ambos o sexos, foram submetidos a uma avaliação antropométrica (IMC e Pregas Cutâneas) e a uma avaliação da capacidade funcional, através de reprodução de atividades do dia-a-dia; de acordo com os resultados que obtiveram, o baixo peso foi fortemente associado a uma diminuição da realização das atividades da vida diárias. Contudo este estudo foi realizado numa instituição para idosos de classe média bai$\mathrm{xa}$, em que a maioria deles apresentou baixo peso e que aqueles com dificuldade na locomoção não foram excluídos da pesquisa. Ou seja, os critérios de exclusão dos idosos na realização dos testes foram diferentes dos usados no presente estudo, o que prejudica a comparação dos resultados. 
Em um estudo realizado por Ferruci et al. ${ }^{16}$, envolvendo 3.381 idosos ( $\geq 71$ anos), o baixo desempenho nos testes relacionados aos membros inferiores (equilíbrio, "sentar e levantar" e velocidade de caminhar, somados em um único escore) mostrou associação positiva $(\mathrm{p}<0,01)$ com maior IMC, em análise de regressão ajustada para sexo e idade. Estes dados corroboram com o resultado deste estudo.

Uma outra variável utilizada para avaliar o EN foi a CP, considerada como a medida mais sensível de massa muscular do idoso, sendo superior à circunferência do braço ${ }^{19,28}$, indicando alterações da massa magra que ocorrem com a idade e com a diminuição da atividade. Os resultados do presente trabalho indicaram que os idosos de ambos os sexos aparentemente não apresentaram um comprometimento da massa magra, já que o valor médio encontrado para o sexo masculino foi de $32,86 \mathrm{~cm}$ e para o sexo feminino de $33,31 \mathrm{~cm}$. No entanto, verificou-se uma associação da CP deficiente com elevado risco de quedas, verificado através do teste AF (Gráfico 2). Esta aparente incoerência pode ser explicada pela interferência da obesidade que diminui a sensibilidade do teste.

Por ser um estudo transversal, os resultados encontrados representam o momento da vida dos idosos sem, portanto, identificar os fatores que conduziram ao observado. Estudos longitudinais são necessários para identificação de causa e efeito.

No presente estudo, a obesidade parece ser o fator predisponente para as associações observadas entre EN e os testes de DM. A associação entre a obesidade e o baixo desempenho na realização dos testes motores não exclui a possibilidade de interferência de outros fatores, como, por exemplo, doenças crônicas e nível de atividade física, que não foram analisados neste estudo.

\section{Conclusões}

Os resultados do presente estudo mostram associação entre o EN e a CF entre os idosos independente do sexo; tendo sido a obesidade apontada como a condição nutricional limitante ao desempenho adequado nos testes. Desta forma, a manutenção da CF, bem como dos níveis normais do IMC podem ser fundamentais para melhorias na qualidade de vida dos idosos.

Tendo em vista os resultados encontrados neste estudo, recomendam-se outras investigações, analisando a associação do estado nutricional com testes que avaliem o desempenho motor, estudos com um número maior de idosos e que utilizem outras variáveis antropométricas, além da realização de estudos longitudinais.

\section{Colaboradores}

LDA Soares, FACS Campos, MGR Araújo, APST Falcão, BRDA Lima, DF Siqueira, EOS Fittipaldi, SGB Arruda e ZP Faro participaram igualmente de todas as etapas de elaboração do artigo. 


\section{Referências}

1. Carvalho JAM, Garcia RA. O envelhecimento da população brasileira: um enfoque demográfico. Cad Saude Publica 2003; 19(3):725-733.

2. Carvalho JAM, Rodriguez-Wong LL. A transição da estrutura etária da população brasileira na primeira metade do século XXI. Cad Saude Publica 2008; 24(3):597-605.

3. Siqueira RL, Botelho MIV, Coelho FMG. A velhice: algumas considerações teóricas e conceituais. Cien Saude Colet 2002; 7(4):899-906.

4. Chaimowicz F. A saúde dos idosos brasileiros às vésperas do século XXI: problemas, projeções e alternativas. Rev Saude Publica 1997; 31(2):184-200.

5. Omran ML, Morley JE. Assessment of protein energy malnutrition in older persons, part I: history, examination, body composition, and screening tools. Nutrition 2000; 16(1):50-63.

6. Fundação Instituto Brasileiro de Geografia e Estatística (IBGE). Pesquisa nacional por amostra em domicílio 2006. Rio de Janeiro: IBGE; 2006.

7. Kalache A, Veras RP, Ramos LR. O envelhecimento da população mundial. Um novo desafio. Rev Saude Publica 1987; 21(3):200-210.

8. Frenk J. Transciones: vidas, instituiciones, ideas. Salud Pública de México 1997; 39(2):144-150.

9. Hamilton IS. A psicologia do envelhecimento: uma introdução. Porto Alegre: Artmed; 2002.

10. Zimerman G. Velhice: Aspectos Biopsicossociais. São Paulo: Artes Médicas; 2000.

11. Gallahue DL, Ozmun JC. Compreendendo o desenvolvimento motor: bebês, crianças, adolescentes e adultos. São Paulo: Phorte; 2002.

12. Guccione AA. Fisioterapia geriátrica. $2^{\text {a }}$ ed. Rio de Janeiro: Guanabara Koogan; 2000.

13. Frontera R, Larsson L. Função da musculatura esquelética nas pessoas idosas. In: Frontera R, Larsson L. Manual de Reabilitação Geriátrica. 1ª ed. Rio de Janeiro: Editora Guanabara Koogan; 2001.

14. Apovian CM, Frey CM, Wood GC, Rogers JZ, Still $\mathrm{CD}$, Jensen GL. Body mass index and physical function in older women. Obes Res. 2002; 10(8):740-747.

15. Bannermann E, Miller MD, Daniels LA, Cobiac L, Giles LC, Whitehead C, Andrews GR, Crotty M. Anthropometric indices predict physical function and mobility in older Australians: the Australian Longitudinal Study of Ageing. Public Health Nutr 2002; 5(5):655-662.

16. Ferrucci L, Penninx BW, Leveille SG, Corti MC, Pahor M, Wallace R, Harris TB, Havlik RJ, Guralnik JM. Characteristics of nondisabled older persons who perform poorly in objective tests of lower extremity function. J Am Geriatr Soc 2000; 48(9):1102-1110.

17. Parahyba MI, Melzer D. Profile of disability in older people in Brazil: results of the PNAD survey. In: Anais do $13^{\circ}$ Encontro nacional de estudos populacionais; 2002; Ouro Preto. Belo Horizonte: ABEP; 2002. (Disponível em CD-ROM)

18. Sociedade Brasileira de Cardiologia, Sociedade Brasileira de Hipertensão, Sociedade Brasileira de Nefrologia. V Diretrizes Brasileiras de Hipertensão Arterial. [site na Internet]. São Paulo: SBC, SBH, SBN; 2006. [acessado 2012 abr 10]. Disponível em: http:/ /publicacoes. cardiol.br/consenso/2006/VDiretrizHA.asp
19. Waitzberg DL, Ferrini MT. Exame Físico e Antropometria. In: Waitzberg DL, organizador. Nutrição oral, enteral e parenteral na prática clínica. 3a ed. São Paulo: Atheneu; 2000. p.255-278.

20. Hautier C, Bonnefoy M. Training for older adults. Ann Readapt Med Phys 2007; 50(6):475-479.

21. Kauffman TL. Manual de Reabilitação Geriátrica. Rio de Janeiro: Guanabara Koogan; 2001.

22. Podsiadlo D, Richardson S. The timed "Up \& Go": a test of basic functional mobility for frail elderly persons. J Am Geriatr Soc 1991; 39(2):142-148.

23. Freitas EV, Miranda RD, Nery MR. Parâmetros Clínicos do Envelhecimento e Avaliação Geriátrica Global. In: Freitas EV, Py L, Neri AL, Cançado FAX, Gorzoni ML, Rocha SM, organizadores. Tratado de Geriatria e Gerontologia. Rio de Janeiro: Guanabara Koogan; 2002. p. 609-617.

24. Duncan PW, Weiner DK, Chandler J, Studenski S. Functional reach: a new clinical measure of balance. J Gerontol 1990; 45(6):192-197.

25. Vellas BJ, Wayne SJ, Romero L, Baumgartner RN, Rubenstein LZ, Garry PJ. One-Leg Balance Is an Important Predictor of Injurious Falls in Older Persons. J Am Geriatr Soc 1997; 45(6):735-738.

26. Cicuttini FM, Teichtahl AJ, Wluka AE, Davis S, Strauss BJ, Ebeling PR. The relationship between body composition and knee cartilage volume in healthy middle-aged subjects. Arthritis Rheum 2005; 52(2):461-467.

27. Tavares EL, Anjos LA. Perfil antropométrico da população brasileira: Resultados da pesquisa nacional sobre saúde e nutrição. Cad Saude Publica 1999; 15(4):759-768.

28. WHO Expert Commitee on physical status. The use and interpretation of antropometry physical status: the use and interpretation of antropometry. Geneve: Report of a WHO Expert Commitee; 1995.

29. Barbosa AR, Souza JMP, Lebrão ML, Marucci MFN. Estado nutricional e desempenho motor de idosos de São Paulo. Revista da Associação Médica Brasileira 2007; 53(1):75-79.

30. Visser M, Deeg DJ, Lips P, Harris TB, Bouter LM. Skeletal muscle mass and muscle strength in relation to lower-extremity performance in older men and women. J Am Geriatr Soc 2000; 48(4):381-386.

31. Lebrão ML, Laurenti R. Saúde, bem-estar e envelhecimento: O estudo SABE no Município de São Paulo. Rev Brasileira de Epidemiologia 2005; 8(2):127141.

32. Maciel ACC, Guerra RO. Limitação funcional e sobrevida em idosos de comunidade. Rev. Assoc. Med. Bras. 2008; 54(4):347-352.

33. Rauen MS, Moreira EAM, Calvo MCM, Lobo AS. Avaliação do estado nutricional de idosos institucionalizados. Rev. Nutrição 2008; 21(3):303-310.

34. Rogatto GP, Gobbi S. Efeitos da atividade física regular sobre parâmetros antropométricos e funcionais de mulheres jovens e idosas. Rev. Brasileira de Cineantropometria e Desempenho Humano 2001; 3(1): 63-69. 
35. Alves RV, Mota J, Costa MC, Alves JGB. Aptidão física relacionada à saúde de idosos: Influência da hidroginástica. Revista Brasileira de Medicina do Esporte 2004; 10(1):31-37.

36. Zuliani G, Romagnoni F, Volpato S, Soattin L, Leoci V, Bollini MC, Buttarello M, Lotto D, Fellin R. Nutritional Parameters, Body Composition, and Progression of Disability in Older Disabled Residents Living in Nursing Homes. Journal of Geronto$\log y$ 2001; 56(4):m212-216

Artigo apresentado em 21/03/2011

Aprovado em 29/06/2011

Versão final apresentada em 11/07/2011 